\title{
Transcriptomic analysis of the ion channelome of human platelets and megakaryocytic cell lines
}

\author{
Joy R. Wright ${ }^{*}, 3$; Stefan Amisten ${ }^{2 *}$; Alison H. Goodall3; Martyn P. Mahaut-Smith ${ }^{1}$ \\ ${ }^{1}$ Department of Molecular and Cell Biology, University of Leicester, Leicester, UK; ${ }^{2}$ Division of Diabetes and Nutritional Sciences, Kings College, London, UK; ${ }^{3}$ Department of \\ Cardiovascular Sciences, University of Leicester and NIHR Cardiovascular Biomedical Research Unit, Glenfield Hospital, Leicester, UK
}

\begin{abstract}
Summary
Ion channels have crucial roles in all cell types and represent important therapeutic targets. Approximately 20 ion channels have been reported in human platelets; however, no systematic study has been undertaken to define the platelet channelome. These membrane proteins need only be expressed at low copy number to influence function and may not be detected using proteomic or transcriptomic microarray approaches. In our recent work, quantitative real-time PCR (qPCR) provided key evidence that Kv1.3 is responsible for the voltage-dependent $\mathrm{K}^{+}$conductance of platelets and megakaryocytes. The present study has expanded this approach to assess relative expression of 402 ion channels and channel regulatory genes in human platelets and three megakaryoblastic/erythroleukaemic cell lines. mRNA levels in platelets are low compared to other blood cells, therefore an improved method of isolating platelets was developed. This used a cocktail of inhibitors to prevent formation of leukocyte-platelet aggregates, and a
\end{abstract}

Correspondence to:

Prof. Martyn Mahaut-Smith, PhD

Department of Molecular and Cell Biology

Henry Wellcome Building, University of Leicester

Leicester, LEI 7RH, UK

Tel.: +44 1162297135

E-mail:mpms1@le.ac.uk

* J.R. Wright and S. Amisten contributed equally to this study.

Supplementary Material to this article is available online at

www.thrombosis-online.com. combination of positive and negative immunomagnetic cell separation, followed by rapid extraction of mRNA. Expression of 34 channel-related transcripts was quantified in platelets, including 24 with unknown roles in platelet function, but that were detected at levels comparable to ion channels with established roles in haemostasis or thrombosis. Trace expression of a further 50 ion channel genes was also detected. More extensive channelomes were detected in MEG-01, CHRF-288-11 and HEL cells (195, 185 and 197 transcripts, respectively), but lacked several channels observed in the platelet. These "channelome" datasets provide an important resource for further studies of ion channel function in the platelet and megakaryocyte.

\section{Keywords}

Platelet physiology, megakaryocytes, molecular biology methods, receptors

\section{Financial support:}

We acknowledge support of the British Heart Foundation (grant no PG/11/56) and the Leicester NIHR Biomedical Research Unit in Cardiovascular Disease, Glenfield Hospital, Leicester, UK.

Received: November 19, 2015

Accepted after major revision: April 30, 2016

Epub ahead of print: June 9, 2016

http://dx.doi.org/10.1160/TH15-11-0891

Thromb Haemost 2016; 116: 272-284

\section{Introduction}

Platelets play an essential role in haemostasis and thrombosis and contribute to immune responses, angiogenesis, lymph vessel development and liver regeneration (1). Platelets also have a pathophysiologic role in cancer progression and development of atherosclerosis. Ion channels are transmembrane proteins with ubiquitous roles in homeostasis and cellular activation. Approximately 20 ion channels have been previously reported in platelets or their nucleated precursor cell, the megakaryocyte (2). Whereas some channels, such as ATP-gated P2X1 channels and store-operated Orail channels, have defined roles in platelet activation, the importance of many others remains unclear. Furthermore, since ion channel proteins need only be present at low density to exert a significant influence on cell function, it is likely that additional important members of this protein superfamily are expressed in the platelet and to date remain undetected. For example, each platelet has been estimated to express only 150-300 functional P2X1 receptors (3), compared to approximately 50,000-80,000 copies of the integrin $\alpha_{I I b} \beta_{3}$ (GPIIbIIIa) (4), yet this cation channel is a key contributor to thrombosis in small arteries and arterioles (5).

Proteomics is an important tool that has recently been used to identify $\sim 4000$ proteins in resting platelets from healthy donors (6, 7). Despite the large number of proteins reported, relatively few are ion channels or channel regulatory proteins (21 and 27 in these two studies). This result may reflect the difficulty of visualising proteins with multiple transmembrane domains, such as ion channels, using standard proteomics methods (8). Although anucleate, platelets contain mRNA, therefore an alternative approach to identify platelet proteins is via screening of the transcriptome. Over the last decade, gene expression microarray studies have reported an expanding list of transcripts within the platelet $(9,10)$. 
More recently, next-generation sequencing techniques have identified in excess of 9000 genes expressed in human platelets $(11,12)$. However, even when detection thresholds and correction for false discovery rates have been rigorously applied, qPCR is still required for validation of gene expression and estimation of transcript abundance from RNA-seq reads. Recent qPCR screening of platelet samples for all known voltage-gated $\mathrm{K}^{+}$channel alpha subunits was a key step in the identification of Kv1.3 (gene symbol: KCNA3) as the pore-forming subunit of the voltage-dependent $\mathrm{K}^{+}$ conductance in platelets and megakaryocytes (13). In the present study, we have extended this approach to include 402 genes encoding ion channels and ion-channel-related proteins, with the aim of defining the platelet ion channelome. qPCR was also used to define the channelome of two human megakaryoblastic celllines (MEG-01 and CHRF-288-11), and a human erythroleukaemic/ megakaryocytic cell-line (HEL) (14) which are often used as surrogates for electrophysiological studies of the small, fragile platelet. This is the first study to focus specifically on determining the platelet ion channelome. The data highlight that platelets express mRNA transcripts encoding multiple ion channels and channel regulatory proteins with no known function in this cell type. The study also provides a reference atlas for future studies of this large family of proteins in megakaryocytic cell lines.

\section{Materials and methods \\ Blood collection and platelet purification}

Blood was collected with informed consent from healthy adult donors, as approved by the University of Leicester Human Biology Ethics committee (non-NHS). For each sample, $10 \mathrm{ml}$ of whole blood was drawn into acid-citrate dextrose anticoagulant (ACD; $85 \mathrm{mM}$ trisodium citrate, $78 \mathrm{mM}$ citric acid and $111 \mathrm{mM}$ glucose) and $1.5 \mathrm{ml}$ of a platelet inhibitor cocktail was added immediately to prevent platelet activation ( $2 \mathrm{mM} \mathrm{EDTA}, 0.1 \mu \mathrm{M} \mathrm{PGE}_{1}$ and 0.3 $\mathrm{mM}$ acetylsalicylic acid). Platelet-rich plasma (PRP) was prepared by centrifugation for 20 minutes at room temperature at $150 \times g$, and the upper $80 \%$ was transferred to a clean tube to avoid transfer of leukocytes from the buffy coat. Contamination of PRP by leukocytes or platelet-leukocyte aggregates was further reduced by immunomagentic depletion of CD45+ve cells (Dynabeads ${ }^{\circledR}$, Invitrogen, Paisley, UK). Platelets were then positively selected with Pan mouse IgG Dynabeads ${ }^{\circ}$ (Invitrogen) coated with mouse antihuman CD42b (Becton Dickinson, Oxford, UK). Following removal of the plasma supernatant, the bead-bound platelets were washed in sterile phosphate-buffered saline (PBS), and lysed in Lysis/Binding buffer (100 mM TrisHCl (pH 7.5); $500 \mathrm{mM} \mathrm{LiCl;} 10$ mM EDTA; 1\% LiDS; 5 mM DTT).

\section{Cell culture}

MEG-01, CHRF-288-11, and HEL cell lines were obtained from the European Collection of Cell Cultures (ECACC, Salisbury, UK) and were cultured in RPMI-1640 medium containing $10 \%$ fetal calf serum (Invitrogen), supplemented with $100 \mu \mathrm{g} / \mathrm{ml}$ penicillin and $100 \mu \mathrm{g} / \mathrm{ml}$ streptomycin at $37^{\circ} \mathrm{C}$ in a humidified incubator with $5 \% \mathrm{CO}_{2}$. A total of $4 \times 10^{6}$ cells from each cell line were pelleted by centrifugation, washed in sterile PBS, and lysed in Lysis/ Binding buffer.

\section{Quantitative real-time PCR}

mRNA was directly isolated from all cells using Oligo- $\mathrm{dT}_{25}$ Dynabeads ${ }^{\circ}$ (Invitrogen). The mRNA-bound beads were washed in Tris buffer solution with or without Lithium Dodecyl-Sulfate (LiDS)(Buffer A- $10 \mathrm{mM}$ TrisHCl (pH 7.5); $0.15 \mathrm{M} \mathrm{LiCl;} 1 \mathrm{mM}$ EDTA; 0.1\% LiDS; Buffer B- 10 mM TrisHCl (pH 7.5); $0.15 \mathrm{M}$ $\mathrm{LiCl} 1 \mathrm{mM}$ EDTA), and resuspended in RNase-free water (Sigma, Dorset, UK). mRNA was reverse-transcribed using cloned AMV Reverse Transcriptase (Invitrogen). cDNA was stored at $-80^{\circ} \mathrm{C}$ until required.

Quantitative-PCR analysis was carried out using QuantiFast SYBR green PCR (Qiagen, Manchester, UK) and QuantiTect primer assays (Qiagen) on a 7900HT sequence detection system analyser (ABI Prism, Invitrogen, UK). The genes included in the human ion channelome were identified through manual searches including PubMed (http://www.ncbi.nlm.nih.gov/pubmed); International Union of Basic and Clinical Pharmacology database (IUP HAR;http://www.iuphar-db.org/), Ingenuity Pathways Analysis (http://www.qiagen.com). All primer assays used in the study are listed in Suppl. Table 1 (available online at www.thrombosis-on line.com). Each qPCR included amplification of the housekeeping gene GAPDH and cell-specific genetic markers CD45 (PTPRC)(leukocytes), GYPA (erythrocytes) and GPIBB (platelets), and cycling profiles were applied as per the manufacturer's instructions. Melt curve analysis was performed to confirm specificity of all primers, and specificity was further validated by agarose gel electrophoresis as described elsewhere (15). Only qPCR amplification products matching the theoretical amplification size supplied by Qiagen were assigned as positive. The $\Delta \mathrm{Ct}$ method was used to calculate ion channel gene expression levels, which were then normalised relative to expression of GAPDH. Results are shown as the mean relative gene expression \pm SEM. The platelet channelome results are shown as the mean of data from four separate donors. Channelome results for cell-lines are based on three separate experiments per cell type.

\section{Results \\ Optimisation of platelet purification}

Whilst qPCR is recognised as one of the most sensitive methods for detection of expressed transcripts, previous studies have highlighted the low mRNA content of platelets and thus the possibility of compromised data due to contamination of mRNAs from other blood cells. Furthermore, mRNA can be rapidly degraded, which represents a problem for detection of genes expressed at low levels. Therefore, our initial aim was to improve upon previously reported approaches for platelet purification and cDNA library generation. A combination of inhibitors (see Methods) reduced the 
possibility of platelet activation leading to leukocyte-platelet aggregates. Although Pall leukocyte depletion filters represent a useful approach to remove leukocytes and possible leukocyte-platelet aggregates (15), we found that they substantially reduced the platelet yield ( Figure 1A). In contrast, leukocyte depletion using CD45-coated magnetic beads did not significantly affect platelet number ( Figure 1A). Following leukocyte depletion, the proportion of platelets positively recovered using anti-CD42b-coated magnetic beads increased with bead density ( Figure $1 \mathrm{~B}$ ), reaching $\approx 75 \%$ recovery at the level ( $200 \mu$ lof beads per $1.5 \mathrm{ml}$ of PRP) used subsequently in this study. Following wash steps to remove plasma components, mRNA was directly extracted using Oligo$\mathrm{dT}_{25}$ beads and immediately reverse transcribed, thereby limiting the time available for mRNA degradation. $\mathrm{qPCR}$ of transcripts encoding cell-specific markers for platelets (GPIBB), leukocytes
(CD45), and erythrocytes (GYPA) demonstrated the high purity of platelets in our preparations ( Figure 1C). Leukocyte and erythrocyte samples, obtained from the buffy coat and red cell pellets respectively, provided positive controls for the CD45 and GYPA assays ( $\triangleright$ Figure $1 \mathrm{D})$.

\section{Quantification of expression of the platelet channelome}

qPCR screening of our human platelet cDNA library to identify the 'channelome' resulted in the detection of 84 ion channel or ion channel-related transcripts from a total of 402 genes. Of these, it was possible to provide a rank order of mean expression of 34 genes ( Table 1), with 31 of these genes being expressed in at least three of the four donors, and three (CLCN4, FXYD1, and

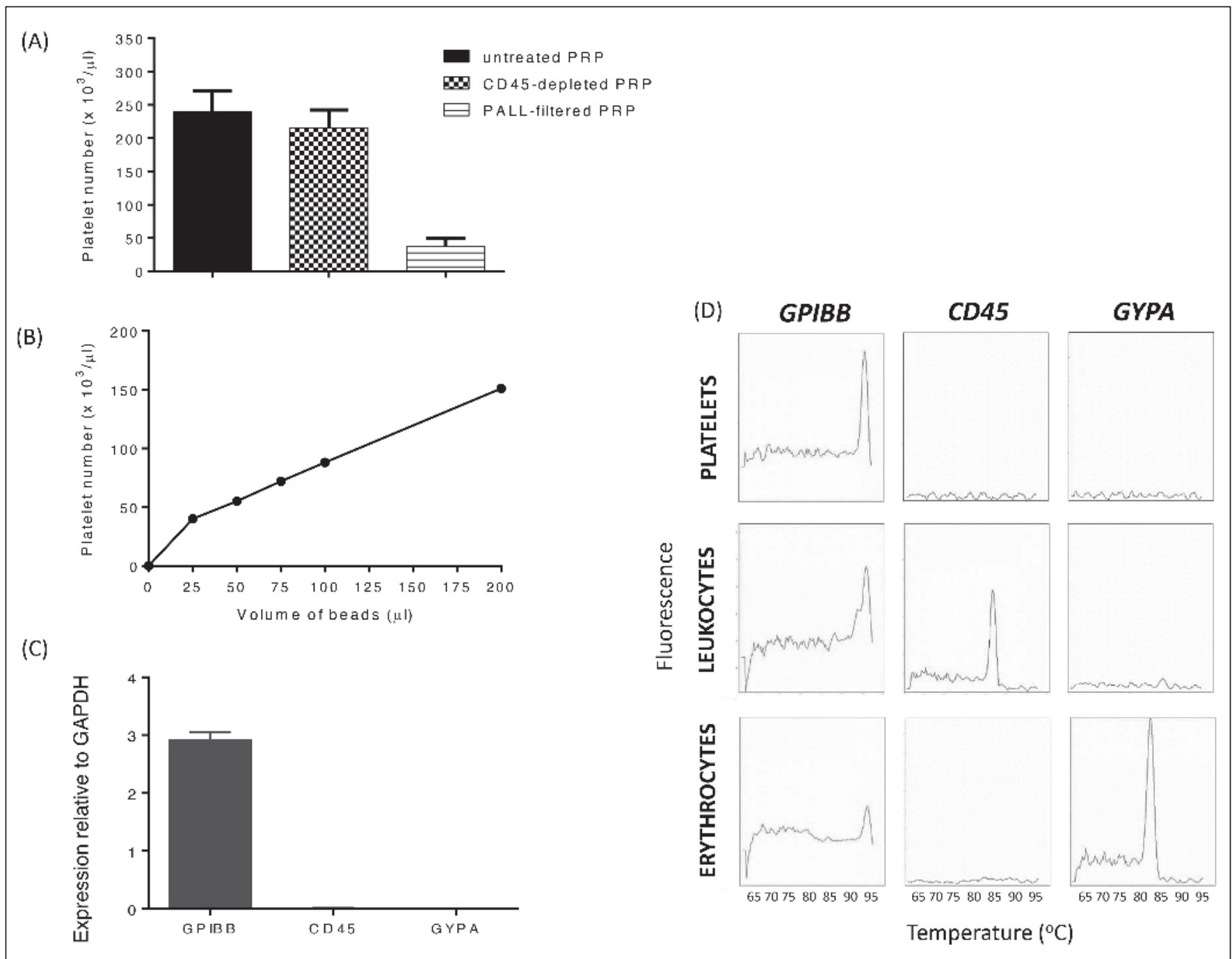

Figure 1: Isolation of ultrapure platelet mRNA. A) Leukocyte depletion using CD45-coated magnetic beads resulted in only minimal loss of platelets from PRP in comparison to leukocyte depletion using PALL filtration. B) Increasing volumes of CD42b-coated magnetic beads enabled positive selection of $\sim 75 \%$ of platelets from $1.5 \mathrm{ml}$ of PRP. C) To test for purity of platelet sample preparations, platelet mRNA was reverse transcribed and amplified for detection of cell-specific markers, GPIBB (platelets), CD45 (PTPRC) (leukocytes) and GYPA (erythrocytes), and expressed relative to the endogenous reference gene GAPDH.D) Melt curve analysis for platelet (GPIBB), leukocyte (CD45) and erythrocyte (GYPA) in platelet sample preparations (top row) used for channelome screening, and in leukocyte (middle row) and erythrocyte (bottom row) sample preparations for comparison. 
ANO10) being quantifiable in two of the four donors. The remaining 50 ion channel transcripts were detected at lower levels, outside of the range of accurate, linear amplification for the qPCR primer assays, and were thus designated as being present only at trace levels ( $\triangleright$ Table 2). Throughout the results section, when referring to platelets, quantifiable genes are defined as GENE NAME (q) and those detected at trace levels as GENE NAME (t). Only 10 of the 84 transcripts detected (11.4\%) have been functionally characterised in platelets or megakaryocytes [ANO6 (alternatively named TMEM16F)(q), P2XR1 (q), KCNA3 (q), CLIC1 (q), ORAI1 (q), PANX1 (q), STIM1 (q), TRPC6 (q), ITPR1 (q) and ITPR2 (q)], and have known agonist-evoked roles in haemostasis or thrombosis $(2,16)$. Seventy-five detected transcripts encoded pore-forming proteins and included 31 voltage-gated channels, 17 ligand-gated channels, and 23 other channel types, as classified by the IUPHAR database, whilst 13 transcripts encoded regulatory proteins or subunits. These families are discussed further below.

\section{The channelomes of three leukaemic myeloid cell lines}

A greater number of ion channel transcripts were detected in all three cell lines compared to the platelet $(185,197$ and 195 for CHRF-288-11, HEL and MEG-01 respectively, vs 84 for the platelet) ( Figure 2A). Differences in abundance of GAPDH transcripts make it difficult to compare the relative expression levels per se between the cell lines and the platelets. However, it is clear that the ion channel expression profiles in these cell lines are very different to that of the platelet, with only 53 transcripts being detected in all four of the cell types ( $>$ Figure 2B). $>$ Table 3 lists the top 20 most abundantly expressed transcripts detected in the cell lines, and a complete list of the relative expression for all channel transcripts included in the study is provided in the Suppl. Table 1 (available online at www.thrombosis-online.com).

\section{Chloride channels show prominent expression in the platelet}

Nine of the quantifiable platelet transcripts, including the four most abundant, encode chloride-permeable ion channels. These include four members of the Anoctamin/TMEM16 family and one member of the Bestrophin family (BEST3)(q). A crucial role for ANO6 (q), the second most abundantly expressed transcript in platelets, has been firmly established in calcium-dependent exposure of phosphatidylserine (PS) on the platelet surface and may contribute to regulation of platelet volume and generation of platelet microparticles in response to haemostatic agonists (16-19). The exact mechanisms whereby ANO6 contributes to these processes remains to be elucidated. In Ano6-deficient mice, significant platelet scramblase activity remains (16), and the underlying proteins for this residual response are not known. Since chloride channel blockers have been reported to block PS exposure in platelets (20), the anion-permeable channels in the platelet channelome list provide a number of possible candidates; however, it is currently unclear whether the other platelet anoctamin channels detected, $A N O 2(\mathrm{q}), A N O 8(\mathrm{t})$ and $A N O 10(\mathrm{q})$, have scramblase activity (17). Interestingly, ANO2, ANO6, ANO8 and ANO10 were recently identified in murine platelets with the same order of expression as the present study (21). Our channelome analysis demonstrates that whereas detection of $A N O 2$ was platelet-specific, ANO6, ANO8 and ANO10 were also expressed in all three cell lines (Suppl. Table 1, available online at www.thrombosis-online. com).

We also detected platelet transcripts for the chloride intracellular channels CLIC1(q), CLIC3(t) and CLIC4(q). CLIC proteins can exist in both soluble and membrane-inserted forms and also exhibit enzymatic activity (22). While there have been no platelet studies identifying the role of CLIC3 or the most abundantly expressed transcript, CLIC4, absence of CLIC1 in mice has been associated with a prolonged bleeding time and a reduced platelet response to ADP (23). These channels may regulate platelet function by affecting ion flow through intracellular organellar membranes; however, they may also become inserted into the plasma membrane following platelet activation, as shown for CLIC1 in platelets (23). We additionally detected transcripts from the CLCN (CIC) family which have been associated with endosomal acidification and trafficking of vesicles (24), finding CLCN3(q), CLCN4(q), CLCN6 $(\mathrm{t})$ and $C L C N 7(\mathrm{t})$ in platelets and in all three cell lines. Voltage-dependent anion channels (VDACs) were also expressed at high levels in platelets and all cell lines. VDACs are located on the outer mitochondrial membrane, regulating the exchange of metabolites between the cytosol and the mitochondrial intermembrane space. Mitochondrial ion channels are key components of the intrinsic apoptosis pathway and contribute to platelet lifespan and cancer cell survival; however, the role of VDACs in platelet biology is unknown. We detected high expression of isoforms $V D A C 2(\mathrm{q})$ and $V D A C 3(\mathrm{q})$ in platelets, and all three isoforms were expressed in the cell lines. Several of the uncharacterised platelet chloride channels, such as CLIC4, CLIC1, VDAC2 and VDAC3, were also amongst the most prevalent transcripts in the CHRF, HEL and MEG-01 cell lines, thus providing an alternative system for their study.

\section{Potassium channels}

A total of 29 ion channel genes expressed in the platelet encode proteins involved in the transport of potassium ions. Nine of these are known to encode pore-forming domains and 19 to be ancillary subunits that modulate $\mathrm{K}^{+}$channel function. The most abundantly expressed pore-forming potassium channel transcript was for the voltage-gated, shaker-related, pore-forming alpha subunit, Kv1.3 $(K C N A 3)(q)$, which we have previously reported to be responsible for the major potassium conductance of the platelet and maintenance of the resting membrane potential (13). High platelet mRNA levels were also detected for $K C N K 6$ (q), a two-pore potassium channel. This channel is widely expressed, and has been reported at the protein level in platelets $(6,7)$; however, it has not been observed in patch clamp studies, and its role in platelet function remains to be determined. KCa1.1 (KCNMA1)(t), a large conductance calcium-activated potassium channel not previously charac- 
Table 1: Quantified ion channel related transcripts in human platelets. Data represent expression of platelet mRNA transcripts relative to endogenous control. Results are expressed as mean values \pm SEM. Reported platelet function detected in: hP, human platelets; hD, human disease; mKO, murine knock-out; mTG, murine transgenic. MP, microparticle.

\begin{tabular}{|c|c|c|c|}
\hline $\begin{array}{l}\text { Gene } \\
\text { symbol }\end{array}$ & Full name & $\begin{array}{l}\text { Relative expression } \\
( \pm \text { SEM) }\end{array}$ & Reported platelet function? \\
\hline CLIC4 & Chloride intracellular channel 4 & $2.759 \pm 0.555$ & No \\
\hline AN06 & Anoctamin 6 (TMEM16F) & $2.020 \pm 0.632$ & $\begin{array}{l}\text { Scramblase activity hP, MP production } \mathrm{hP} \text { mKO, } \\
\text { Scott syndrome } h D \text {, increased bleeding time } \\
\mathrm{mKO}(16-18,21,62)\end{array}$ \\
\hline VDAC3 & Voltage-dependent anion channel 3 & $0.645 \pm 0.235$ & No \\
\hline CLCN3 & Voltage-sensitive chloride channel 3 & $0.581 \pm 0.161$ & No \\
\hline $\mathrm{P} 2 \mathrm{RX} 1$ & Purinergic receptor $\mathrm{P} 2 \mathrm{X}$, ligand-gated ion channel, 1 & $0.397 \pm 0.202$ & $\begin{array}{l}\text { ATP-gated } \mathrm{Ca}^{2+} \text { influx } \mathrm{hP} \text {, arterial thrombosis } \mathrm{hP} \\
\mathrm{mTG}(3,5,38,63)\end{array}$ \\
\hline FXYD5 & FXYD domain-containing ion transport regulator 5 , dysadherin & $0.345 \pm 0.107$ & No \\
\hline KCNA3 & Voltage-gated potassium channel alpha subunit, Kv1.3 & $0.249 \pm 0.062$ & $\begin{array}{l}\text { Membrane potential hP, increased platelet } \\
\text { count mKO (13) }\end{array}$ \\
\hline CLIC1 & Chloride intracellular channel 1 & $0.241 \pm 0.041$ & Role in ADP-evoked signalling mKO (23) \\
\hline LRRC8B & Leucine-rich repeat-containing protein 8B & $0.202 \pm 0.088$ & No \\
\hline ORAl1 & ORAI calcium release-activated calcium modulator 1 & $0.124 \pm 0.028$ & $\begin{array}{l}\text { Store Operated } \mathrm{Ca}^{2+} \text { Entry (SOCE) hP mKO, } \\
\text { impaired thrombus formation mKO }(32,33)\end{array}$ \\
\hline PANX1 & Pannexin 1 & $0.113 \pm 0.039$ & ATP release $\mathrm{hP}$ mKO $(47,64)$ \\
\hline KCNE3 & Potassium voltage-gated channel, Isk-related family member 3, MiRP2 & $0.092 \pm 0.019$ & No \\
\hline ITPR2 & Inositol 1,4,5-triphosphate receptor, type 2 & $0.088 \pm 0.040$ & Agonist-evoked $\mathrm{Ca}^{2+}$ mobilisation $\mathrm{hP}(2,65)$ \\
\hline STIM1 & Stromal interaction molecule 1 & $0.086 \pm 0.023$ & $\begin{array}{l}\mathrm{Ca}^{2+} \text { sensor for SOCE hP mKO, impaired } \\
\text { thrombus formation mKO }(2,32,66,67)\end{array}$ \\
\hline VDAC2 & Voltage-dependent anion channel 2 & $0.053 \pm 0.016$ & No \\
\hline KCTD20 & Potassium channel tetramerisation domain-containing protein 21 & $0.051 \pm 0.005$ & No \\
\hline TRPC6 & Transient receptor potential cation channel, subfamily C, member 6 & $0.050 \pm 0.030$ & $\begin{array}{l}\mathrm{Ca}^{2+} \text { entry, haemostasis and thrombogenesis } \\
\mathrm{hP} \text { mKO }(2,27,28,68,69)\end{array}$ \\
\hline ANO2 & Anoctamin 2 (TMEM16B) & $0.037 \pm 0.007$ & No \\
\hline KCTD10 & Potassium channel tetramerisation domain-containing protein 10 & $0.036 \pm 0.011$ & No \\
\hline KCNK6 & Potassium channel, subfamily K, member 6; K2p6.1 & $0.035 \pm 0.010$ & No \\
\hline $\mathrm{AQP10}$ & Aquaporin 10 & $0.034 \pm 0.014$ & No \\
\hline CLCN4 & Voltage-sensitive chloride channel 4 & $0.033 \pm 0.004$ & No \\
\hline FXYD1 & FXYD domain-containing ion transport regulator 1, phospholemman & $0.025 \pm 0.012$ & No \\
\hline KCNMB1 & Calcium-activated potassium channel subfamily M, K(VCA)beta-1 & $0.025 \pm 0.011$ & No \\
\hline BEST3 & Bestrophin-3, Vitelliform macular dystrophy 2-like protein-3 & $0.025 \pm 0.002$ & No \\
\hline CHRNA2 & Cholinergic receptor, nicotinic, alpha 2 & $0.023 \pm 0.007$ & No \\
\hline KCTD18 & Potassium channel tetramerisation domain-containing protein 18 & $0.022 \pm 0.003$ & No \\
\hline KCTD2 & Potassium channel tetramerisation domain-containing protein 2 & $0.016 \pm 0.003$ & No \\
\hline KCTD13 & Potassium channel tetramerisation domain-containing protein 13 & $0.016 \pm 0.005$ & No \\
\hline ITPR1 & Inositol 1,4,5-triphosphate receptor, type 1 & $0.015 \pm 0.002$ & Agonist-evoked $\mathrm{Ca}^{2+}$ mobilisation $\mathrm{hP}(2,65,70)$ \\
\hline AN010 & Anoctamin 10 & $0.011 \pm 0.002$ & No \\
\hline KCNMB4 & Calcium-activated potassium channel subunit beta- 4 & $0.009 \pm 0.003$ & No \\
\hline TMEM109 & Transmembrane protein 109; Mitsugumin-23. & $0.007 \pm 0.001$ & No \\
\hline KCTD9 & Potassium channel tetramerisation domain-containing protein 9 & $0.004 \pm 0.001$ & No \\
\hline
\end{tabular}


Table 2: Ion channel-related transcripts detected at trace levels in human platelets.

\begin{tabular}{|c|c|c|c|}
\hline $\begin{array}{l}\text { Gene } \\
\text { symbol }\end{array}$ & Protein name & $\begin{array}{l}\text { Gene } \\
\text { symbol }\end{array}$ & Protein name \\
\hline AN08 & Anoctamin 8 & KCNE2 & Mirp1 \\
\hline $\mathrm{AQP1}$ & Aquaporin 1 (Colton blood group) & KCNG4 & Modifier/silencer; Kv6.4 \\
\hline CACNB1 & Calcium channel, voltage-dependent, beta 1 subunit & KCNK1 & Potassium channel, subfamily K, member 1; K2p1.1, TWIK-1 \\
\hline CATSPER1 & CatSper1; cation channel sperm-associated protein-1 & KCNK17 & Potassium channel, subfamily K, member 17; K2p17.1, TASK4 \\
\hline CLCN6 & Chloride channel 6 & KCNMA1 & $\begin{array}{l}\text { Potassium large conductance calcium-activated channel, subfamily M, alpha } \\
\text { member } 1\end{array}$ \\
\hline CLCN7 & Chloride channel 7 & KCNMB3 & Calcium-activated potassium channel subunit beta-3 \\
\hline CLIC3 & Chloride intracellular channel 3 & KCNN3 & $\begin{array}{l}\text { Potassium intermediate/small conductance calcium-activated channel, } \\
\text { subfamily N, member 3; KCa2.3 }\end{array}$ \\
\hline FXYD7 & FXYD domain-containing ion transport regulator 7 & KCNQ4 & Non-inactivating voltage-gated; Kv7.4 \\
\hline GABRD & Gamma-aminobutyric acid (GABA) A receptor, delta & KCNRG & Potassium channel regulatory protein \\
\hline GABRR2 & Gamma-aminobutyric acid (GABA) A receptor, beta 2 & KCNT2 & Potassium channel, subfamily T, member 2 \\
\hline GJA4 & Gap junction protein, alpha $4,37 \mathrm{kDa}$ & KCTD11 & Potassium channel tetramerization domain-containing protein 11 \\
\hline GJB4 & Gap junction protein, beta $4,30.3 \mathrm{kDa}$ & KCTD5 & Potassium channel tetramerization domain-containing protein 5 \\
\hline GJB5 & Gap junction protein, beta 5, 31.1kDa & KCTD7 & Potassium channel tetramerization domain-containing protein 7 \\
\hline GLRA2 & Glycine receptor, alpha 2 & ORAI2 & ORAI calcium release-activated calcium modulator 2 \\
\hline GLRA4 & Glycine receptor, alpha 4 & P2RX4 & Purinergic receptor $\mathrm{P} 2 \mathrm{X}$, ligand-gated ion channel, 4 \\
\hline GLRB & Glycine receptor, beta & P2RX6 & Purinergic receptor P2X, ligand-gated ion channel, 6 \\
\hline GRIK4 & Glutamate receptor, ionotropic, kainate 4 & PIEZO1 & Fam38A; Piezo-type mechanosensitive ion channel component \\
\hline GRIK5 & Glutamate receptor, ionotropic, kainate 5 & PKD1 & Polycystin-1; TRPP1 \\
\hline GRIN2D & $\begin{array}{l}\text { Glutamate receptor, ionotropic, N-methyl D-aspartate } \\
\text { 2D }\end{array}$ & SCN1B & Sodium channel, voltage-gated, type I, beta \\
\hline GRINA & $\begin{array}{l}\text { Protein lifeguard 1; Glutamate [NMDA] receptor- } \\
\text { associated protein } 1\end{array}$ & $\mathrm{SCN} 3 \mathrm{~B}$ & Sodium channel, voltage-gated, type III, beta subunit \\
\hline HVCN1 & Hydrogen voltage-gated channel 1 & SCN5A & Sodium channel, voltage-gated, type V, alpha subunit; Nav1.5 \\
\hline KCNA2 & $\begin{array}{l}\text { Shaker-related non-inactivating delayed rectifier alpha, } \\
\text { Kv1.2 }\end{array}$ & SCNN1A & Sodium channel, nonvoltage-gated 1 alpha \\
\hline KCNAB1 & Beta subunit shaker-related, Kvbeta1 & STIM2 & Stromal interaction molecule 2 \\
\hline KCNAB2 & Beta subunit shaker-related, Kvbeta2 & STX1B & Syntaxin-1B \\
\hline KCNAB3 & Beta subunit shaker-related, Kvbeta3 & TPCN1 & Two pore segment channel 1 \\
\hline
\end{tabular}

terised in platelets, and three regulatory subunits of KCNMA1 were also detected (KCNMB1(q), KCNMB3(t) and KCNMB4(q)). Amongst the other $\mathrm{K}^{+}$channel regulatory subunit transcripts detected were the $K C N A B 1(\mathrm{t}), K C N A B 2(\mathrm{t})$ and $K C N A B 3(\mathrm{t})$ voltagegated shaker-related subunits, also KCNE2(t) (Mirp1) and KCNE3(q) (Mirp2) from the Isk-related family, and potassium family regulatory protein $(K C N R G)(\mathrm{t})$, which has been reported to suppress voltage-gated $\mathrm{K}^{+}$channel currents in Xenopus oocytes (25). Additionally, we detected nine transcripts encoding potassium channel tetramerisation domain-containing proteins (KCTDs) ( Table 1 and $>$ Table 2). Very little is currently known about this large family of proteins, although some of these proteins have been reported to interact with GABAB GPCRs, resulting in modulation of receptor sensitisation (26). Further studies are required to determine the possible roles these regulatory proteins may play in platelet function.

\section{Calcium-permeable ion channels}

Elevation of cytosolic calcium is essential for activation of most platelet functional responses. Amongst the known $\mathrm{Ca}^{2+}$-permeable channels that we detected in platelets were $P 2 R X 1(\mathrm{q})$, ORAI1(q), TRPC6(q), and the inositol 1,4,5-trisphosphate ( $\left.\mathrm{IP}_{3}\right)$ receptor isoforms, ITPR1(q) and ITPR2(q). This provides further 
evidence that TRPC6 is the main member of the TRP family of non-selective cation channels expressed in platelets, as previously suggested (27). Despite several reports of TRPC3 function in platelets $(28,29)$, we did not detect transcripts for TRPC3 mRNA, although it was detectable in all three cell-lines. Trace levels of TRPP1(t) (also known as PKD1 and polycystin) channels were also detected in platelets. We have previously reported detection and function of TRPM7 in murine primary megakaryocytes (30), and in agreement with this we detected TRPM7 in both megakaryocytic cell-lines; however, we did not detect TRPM7 in platelets. A wider range of TRP channels were detected in the leukaemic lines, with TRPV2 the most highly expressed TRP channel transcript in all three cell lines. This heat- and mechanically-activated channel was not observed in the platelet; however, consistent with this study, it has been implicated in haematologic malignancies (31).

It is well established that platelet store-operated calcium entry (SOCE) is mediated through activation of channels formed by Orail in response to STIM1-mediated detection of reduced calcium content of the dense tubular system (DTS) $(32,33)$. Abundant ORAI1(q) transcripts were detected in platelets and all cell lines, whereas only trace levels of ORAI2(t) mRNA were found in all cell types. ORAI3 transcripts were quantifiable in all three cell lines but were not detected in the platelet. Transcripts for the calcium sensors STIM1(q) and STIM2(t) were present in platelets and all cell lines. Studies in Stim2-KO mice suggest there is normal platelet function and calcium homeostasis in response to GPVI- and thrombin-dependent activation (34). Interestingly, STIM2 has been shown to act as a regulator of basal calcium concentrations in the endoplasmic reticulum (ER) of HeLa cells (35). We also detected TMEM109(q), an organellar membrane protein which may serve as a counterion transport mechanism during $\mathrm{Ca}^{2+}$ store release and has been associated with cell death in murine thymocytes (36).

Agonist binding to platelet surface GPCRs and tyrosine kinasecoupled receptors results in generation of $\mathrm{IP}_{3}$ and thus activation of calcium-permeable $\mathrm{IP}_{3}$ receptors $\left(\mathrm{IP}_{3} \mathrm{Rs}\right)$; however, to date the relative expression of the three $\mathrm{IP}_{3} \mathrm{R}$ isoforms in the platelet remains unclear. We detected type 1 (ITPR1)(q) and type 2 $(I T P R 2)(q) \mathrm{IP}_{3} \mathrm{R}$ mRNAs in platelets, although surprisingly a greater number of transcripts of type $2 \mathrm{IP}_{3} \mathrm{Rs}$ were detected. An alternative pathway of calcium mobilisation from intracellular stores has been suggested to occur via two-pore channels, TPCN1 and TPCN2. Whereas both isoforms were detected in the three cell

(A)

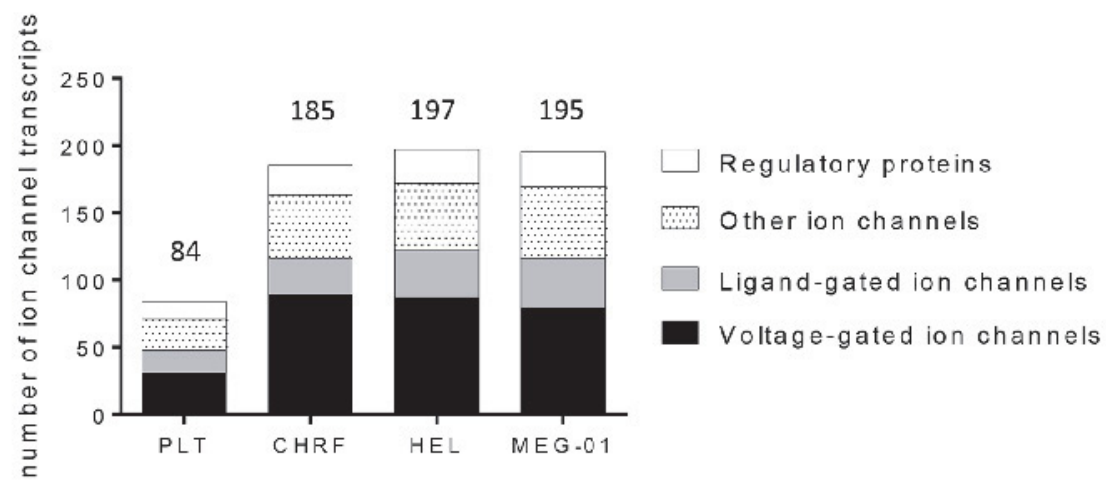

Figure 2: The channelome of platelets and cell-lines. A) Classification of ion channel-related transcripts in platelets and cell lines. The number of ion channel transcripts detected is shown for each cell type, and is further divided into channel type or regulatory protein according to the IUPHAR and UniProt classifications. B) Venn diagram depicting the distribution and overlap of ion channel-related transcripts between platelets, and the cell lines CHRF-288-11, HEL and MEG-01. 
Table 3: Most abundant ion channel-related transcripts in megakaryoblastic and megakaryocytic cell lines. Data represent expression of mRNA transcripts relative to endogenous control. Results are expressed as mean values (see Suppl. Table 1 for SEM, available online at www.thrombosis-online. com). nd, not detected; td, trace detection.

\begin{tabular}{|c|c|c|c|c|}
\hline \multirow{2}{*}{$\begin{array}{l}\text { Gene } \\
\text { symbol }\end{array}$} & \multirow[t]{2}{*}{ Full name } & \multicolumn{3}{|c|}{ Relative expression } \\
\hline & & CHRF & HEL & MEG-01 \\
\hline CLIC1 & Chloride intracellular channel 1 & 0.2338 & 0.0662 & 0.0827 \\
\hline CLNS1A & Chloride conductance, nucleotide sensitive protein $1 \mathrm{~A}$ & 0.1066 & 0.0683 & 0.0750 \\
\hline CLIC4 & Chloride intracellular channel 4 & 0.0606 & 0.0962 & 0.0503 \\
\hline VDAC2 & Voltage-dependent anion channel 2 & 0.0944 & 0.0611 & 0.0315 \\
\hline VDAC3 & Voltage-dependent anion channel 3 & 0.0465 & 0.0412 & 0.0859 \\
\hline FXYD5 & FXYD domain-containing ion transport regulator 5 ; dysadherin & 0.0425 & 0.0675 & 0.0294 \\
\hline TOMM40 & Mitochondrial import receptor subunit homolog; Protein haymaker, p38.5 & 0.0305 & 0.0300 & 0.0568 \\
\hline CLIC2 & Chloride intracellular channel 2 & 0.0005 & 0.0155 & 0.0499 \\
\hline CLCA1 & CLCA family member 1 , chloride channel regulator & nd & nd & 0.0449 \\
\hline GRINA & Protein lifeguard 1; Glutamate [NMDA] receptor-associated protein 1 & 0.0078 & 0.0276 & 0.0254 \\
\hline PKD1 & Polycystin-1; TRPP1 & 0.0262 & 0.0067 & 0.0073 \\
\hline VDAC1 & Voltage-dependent anion channel 1; porin & 0.0021 & 0.0022 & 0.0245 \\
\hline KCTD15 & Potassium channel tetramerization domain-containing protein 15 & 0.0007 & 0.0210 & 0.0116 \\
\hline KCNK17 & Potassium channel, subfamily K, member 17; K2p17.1 & 0.0181 & 0.0041 & $\mathrm{td}$ \\
\hline GJA4 & Gap junction protein, alpha 4, 37kDa & 0.0123 & 0.0013 & $\mathrm{td}$ \\
\hline GJA5 & Gap junction protein, alpha 5, 40kDa & 0.0004 & 0.0015 & $\mathrm{td}$ \\
\hline TMEM38B & TRIC type sub-channel B & 0.0112 & 0.0087 & 0.0118 \\
\hline PIEZO1 & Piezo-type mechanosensitive ion channel component; Fam38A & 0.0051 & 0.0110 & 0.0088 \\
\hline KCNE3 & Potassium voltage-gated channel, Isk-related family, member 3; MiRP2 & 0.0057 & 0.0105 & 0.0008 \\
\hline KCNN4 & $\begin{array}{l}\text { Potassium intermediate/small conductance calcium-activated channel, subfamily N, member 4; } \\
\text { KCa3.1 }\end{array}$ & 0.0011 & 0.0047 & 0.0104 \\
\hline
\end{tabular}

lines, only TPCN1 $(\mathrm{t})$ was detected in platelets. These calcium-permeable ion channels are sensitive to pyridine nucleotides, NAADP, and insert into lysosomal organelles, releasing calcium into the cytoplasm from the acidic stores (37); however, further study is needed to demonstrate that this calcium-activated calcium release channel is functional in the platelet. mRNAs encoding P2X1 receptors $(P 2 R X 1)(\mathrm{q})$ were the fifth most abundantly expressed platelet ion channel transcript. This ATP-gated non-selective cation channel has been well characterised in platelets and shown to contribute to arterial thrombosis $(2,5)$. We found only trace detection of $P 2 R X 4(\mathrm{t})$ and $P 2 R X 6(\mathrm{t})$ transcripts, in agreement with previous work showing that $\mathrm{P} 2 \mathrm{X} 1$ is the dominant ATP-gated $\mathrm{P} 2 \mathrm{X}$ receptor of the platelet. P2RX1 was also expressed in the HEL and MEG-01 cell lines as found previously $(38,39)$, and the cell lines also expressed P2RX4, P2RX5 and P2RX7.

In addition to ATP, platelets also secrete the neurotransmitters glutamate, serotonin and acetylcholine. Functional NMDA, AMPA and kainite ionotropic glutamate receptors have been reported in the platelet and megakaryocyte $(2,40,41)$. We detected components of kainate (GRIK4(t), GRIK5(t)), NMDA $(G R I N 2 D)(\mathrm{t})$ receptors, and GRINA (protein lifeguard 1/NMDA receptor associated protein 1$)(\mathrm{t})$, but not AMPA receptor transcripts in platelets. Whereas it has been established that activation of the platelet GPCR serotonin receptor 5HTR2A results in calcium mobilisation (42), the presence and function of 5HTR3 ion channel family members remains less certain. Our qPCR screen included the 5HTR3 ion channel family of genes, i.e. all known serotonin-gated ion channels, finding no detectable transcripts in human platelets, but quantifiable levels of HTR3D in CHRF, and trace detection of the same gene in HEL and MEG-01 cells. It has been suggested that acetylcholine potentiates the platelet response to ADP and thromboxane A2, influencing early aggregation (2). In the present study we detected mRNA encoding the calcium-permeable a2 nicotinic cholinergic receptor (CHRNA2)(q) only in platelets, whereas the $a 5$ transcript (CHRNA5) was detected only in the cell lines. Other ligand-gated channels detected included GABAA-receptor subunits $(G A B R D(\mathrm{t})$ and $G A B R R 2(\mathrm{t}))$ and glycine receptors ( $\alpha$-subunits GLRA2(t) and GLRA4(4), and $\beta$-subunit $G L R B(\mathrm{t}))$. Ligand-binding of glycine may result in the influx of chloride ions, thereby regulating the platelet membrane potential (43). 


\section{Possible roles of other detected ion channels in the platelet}

Gap junction-mediated intercellular communication allows the brief transfer of small molecules (up to $\approx 1 \mathrm{kDa}$ ). Twenty members of the connexin family have been reported in mammals. We detected Cxn $37(G J A 4)(\mathrm{t})$, which is known to contribute to platelet function $(44,45)$, and also mRNAs for Cxn $30.3(G J B 4)(t)$ and Cxn $31.1(G J B 5)(\mathrm{t})$, not previously reported in platelets. A wider range of connexin transcripts were detected in the cell-lines but only at trace levels. Although similar in structure to connexins, pannexins do not form gap junctions (46); however, the channels (sometimes referred to as hemi-channels) allow rapid permeation of molecules $<1 \mathrm{kDa}$ in size. Three pannexin (PANX) hemi-channel subtypes exist in mammals, but platelets and MEG-01 cells only expressed PANX1(q). Studies in our laboratory have recently demonstrated functional surface pannexin-1 in platelets, demonstrating a mechanism for non-vesicular release of ATP (47). CHRF expressed PANX2, and HEL expressed all three PANX transcripts. Interest-

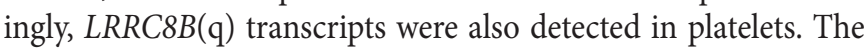
LRRC8 family of proteins (Leucine-rich repeat containing protein 8 ), share sequence and structural homology with the pannexin family (48), and have recently been proposed to be components of a volume-regulated ion channel (VRAC) in human embryonic kidney (HEK) cells (49).

Regulation of cell volume is crucial to maintain the integrity of the cell membrane. One ion channel family, as yet uncharacterised in platelets or megakaryocytes, is the aquaporins. We detected $A Q P 10$ (q), encoding an aqua-glyceroporin, and low levels of $A Q P 1(\mathrm{t})$ in platelets. These transcripts were also detected in the cell lines, which additionally expressed $A Q P 3$ and $A Q P 11$. An increase in cell volume and size results in stretching of the plasma membrane, and this change in membrane tension is detected by mechanosensitive proteins. To date there have been no mechanosensitive ion channels identified in platelets. This study found low levels of transcripts encoding PIEZO1 (FAM38A)(t), reported to be a calcium-permeable mechanically activated cation channel involved in homeostasis of erythrocyte cell volume (50).

In addition to their haemostatic function, platelets also contribute to innate immunity; however, the identities and importance of specific ion channels that contribute to this response remains to be determined. The voltage-gated proton channel, HVCN1(t), detected in platelets and all cell lines, has an established role in supporting superoxide generation via NADPH oxidase, which is known to take place in platelets. Additional contributions to cellular responses have emerged for HVCN1 in recent years, including modulation of immune receptor signalling in B lymphocytes (51). Detection of other channel components in platelets included trace levels of the ion conducting pore-forming alpha-subunit $S C N 5 A(N a v 1.5)(\mathrm{t})$, and two beta subunits, $S C N 1 B$ (beta-1)(t) and SCNB3 (beta-3)(t). Generally associated with expression in cardiac muscle, this sodium channel has also been reported in human monocyte-derived macrophages, regulating endosomal $\mathrm{pH}$ and phagocytosis through attenuation of localised calcium oscillations during exposure to bacterial lipopolysaccharides (52).

\section{Discussion}

Although platelets are known to express a number of ion channels with important functional roles $(5,32,33,38,53,54)$, we hypothesised that additional members of this superfamily of membrane proteins are expressed and may not have been detected in previous studies of the whole platelet proteome or transcriptome. This proposal was based on two main facts: firstly, ion channels need only be expressed at low copy number to influence cell function, and secondly, platelets contain low levels of RNA. Therefore, detection of ion channels amongst more abundantly expressed proteins or transcripts may be problematic. Proteomic and transcriptomic approaches have their advantages and disadvantages, and in addition, there is disagreement as to the degree of correlation between the transcriptome and proteome $(7,9,10)$. Comparison of one RNA-seq study (55), with the findings of the proteomic report from Burkhart et al. (7) suggests that whereas the platelet transcriptome has a high inter-individual correlation, a significant number of transcripts are not translated (55). In contrast, Rowley et al. (56) suggest a much higher correlation between proteome and transcriptome. In support of the latter, a frequency density distribution analysis of platelet transcriptomic and protemic data reported that they are almost identical (57). The on-going debate highlights that there are many factors to be borne in mind when comparing proteomic and transcriptomic datasets, including donor variation, ID mapping, methodological and statistical handling differences.

Whilst recent proteomic studies have made a significant contribution to the characterisation of the platelet proteome $(6,7)$, methods used within the proteomic process may result in the under-representation of transmembrane proteins such as ion channels. One example of this is a recent study of the human proteome which characterised the proteomic signature of several tissues, that reported only five ion channels in the platelet (58). In addition, a common limitation of both proteomic and transcriptomic array studies is the difficultyof directly quantifying low abundance proteins or mRNA transcripts, and also the masking of weak signals by the products of highly expressed genes. Therefore, in this study we have used qPCR and focused only on ion channel-related targets, an approach that we have used previously to successfully identify KCNA3 (Kv1.3) (13) and PANX1 (Pannexin-1) (47) ion channel transcripts in human platelets. These early studies have demonstrated an important role for Kv1.3 in maintenance of the platelet resting membrane potential, and for Pannexin- 1 in the release of non-vesicular ATP leading to amplification of calcium influx. The results of our 'channelome' study of ion channel-related transcripts compare favourably with a RNA-seq study of the whole human platelet transcriptome (11), reporting an overlap in detection of 61 ion channel-related mRNA transcripts from our ion channel database; with ANO6 (TMEM16F) being significantly expressed in both studies. The most abundantly expressed tran- 
Table 4: Estimated channel densities per platelet: electrophysiological and proteomic quantification. MK, megakaryocyte; Plt, platelet; $\mathrm{Cm}$, circumference; pS, picoSiemens; pA, picoamperes; $\mathrm{mV}$, millivolts.

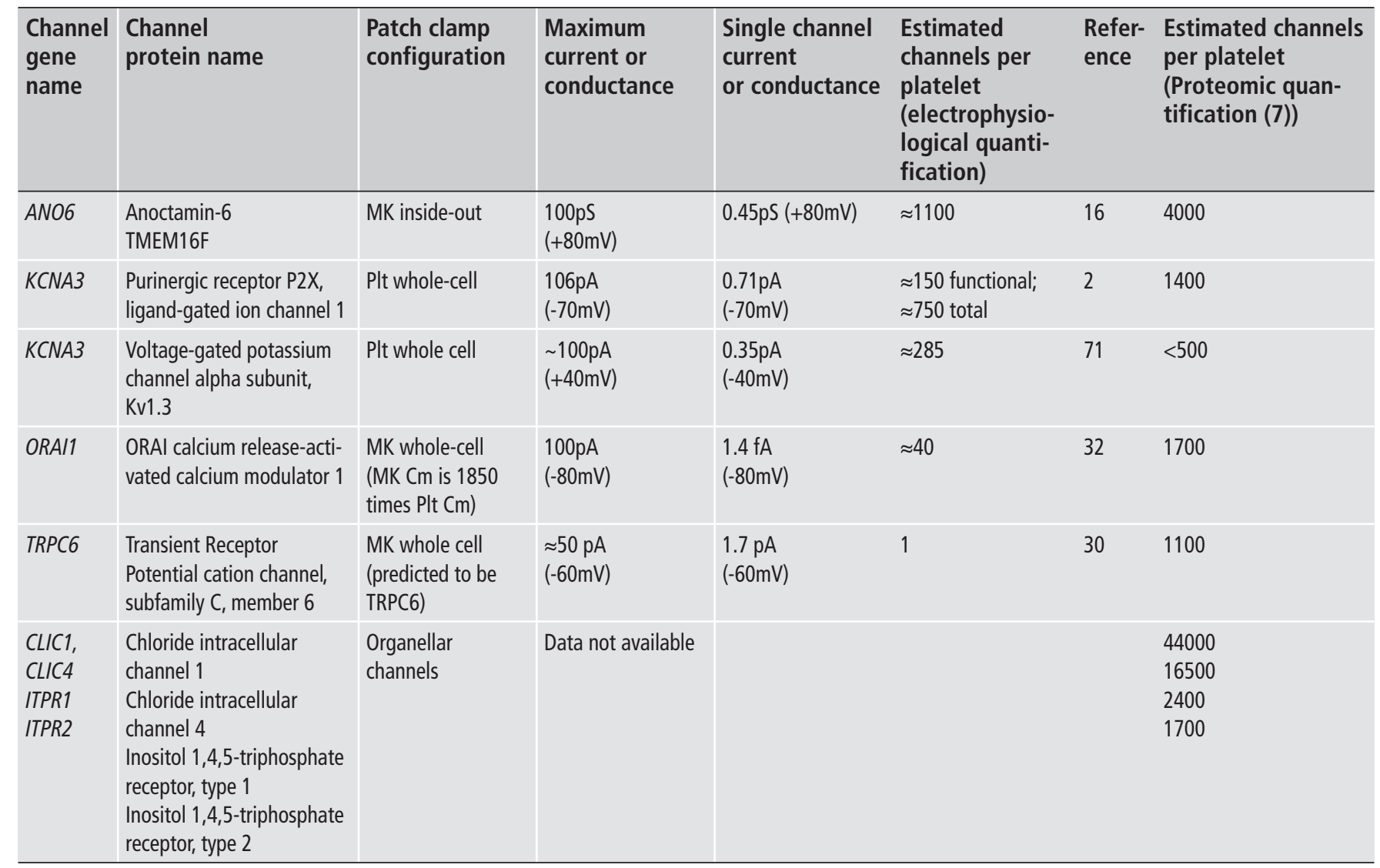

scripts in our study included nine channels previously reported and at least partly characterised in platelets, and the order of their expression correlates closely both with estimations of relative channel density reported in patch clamp recordings, and correlates at least partly with the estimated copy number determined by proteomic quantification (7) ( Table 4).

One of the major sources of platelet mRNA is its precursor cell, the megakaryocyte (MK). During MK maturation, mRNA is packaged into granules and transported into the proplatelet tips and therefore is present in the newly formed platelet. A second possible source of platelet mRNA is that which has been taken up by the platelet, for example from microvesicles that are elevated in the circulation in a range of disease states. The platelets used in this study were collected from healthy donors with no known disease, and therefore the mRNA analysed is likely to reflect a transcriptome generally found in platelets within the circulation of healthy individuals, the source of which is predominantly from the megakaryocyte. Therefore, decay of mRNA, together with the relatively low abundance of mRNA in comparison to other peripheral blood cells must be considered as a possible limitation to transcriptomic study results. Indeed, our previous whole cell patch clamp recordings show that each human platelet possesses only 5-7 functional medium conductance $\mathrm{Ca}^{2+}$ activated $\mathrm{K}^{+}$channels (KCa3.1, the
Gardos channel, gene name KCNN4) (59), but we were unable to detect this extremely low abundance transcript in platelets. In contrast, it was observed in all three leukaemic cell lines (see below), including HEL cells, where robust currents through this channel have been reported in patch clamp studies (60). Interestingly there were 53 ion channel-related transcripts that were only expressed in the three cell lines and not in the platelet, these included TRPC1, TRPC3, and TRPM7, therefore it is possible that some of these channels are also so few in number that they are below the level of detection at the transcriptomic level in this study. Conversely, there were six transcripts detected in platelets that were not present in any of the cell-lines. Of this group, the most abundantly expressed transcript was ANO2 (alternatively named TMEM16B), encoding the second member of the Calciumactivated chloride channels, Anoctamin-2. There are several possible explanations as to why these transcripts were only detected in the platelets and not in the cell lines; including deletion or insertion of additional transcripts during continual culture of cell lines, or reflection of a genotype/phenotype from their cancer cell origin. Of note, Kv1.3 (KCNA3) was absent in CHRF and HEL cell lines and detected only at trace levels in MEG-01. This agrees with a previous report that voltage-dependent $\mathrm{K}^{+}$currents are absent or greatly reduced in megakaryocytic cell lines and in megakaryo- 


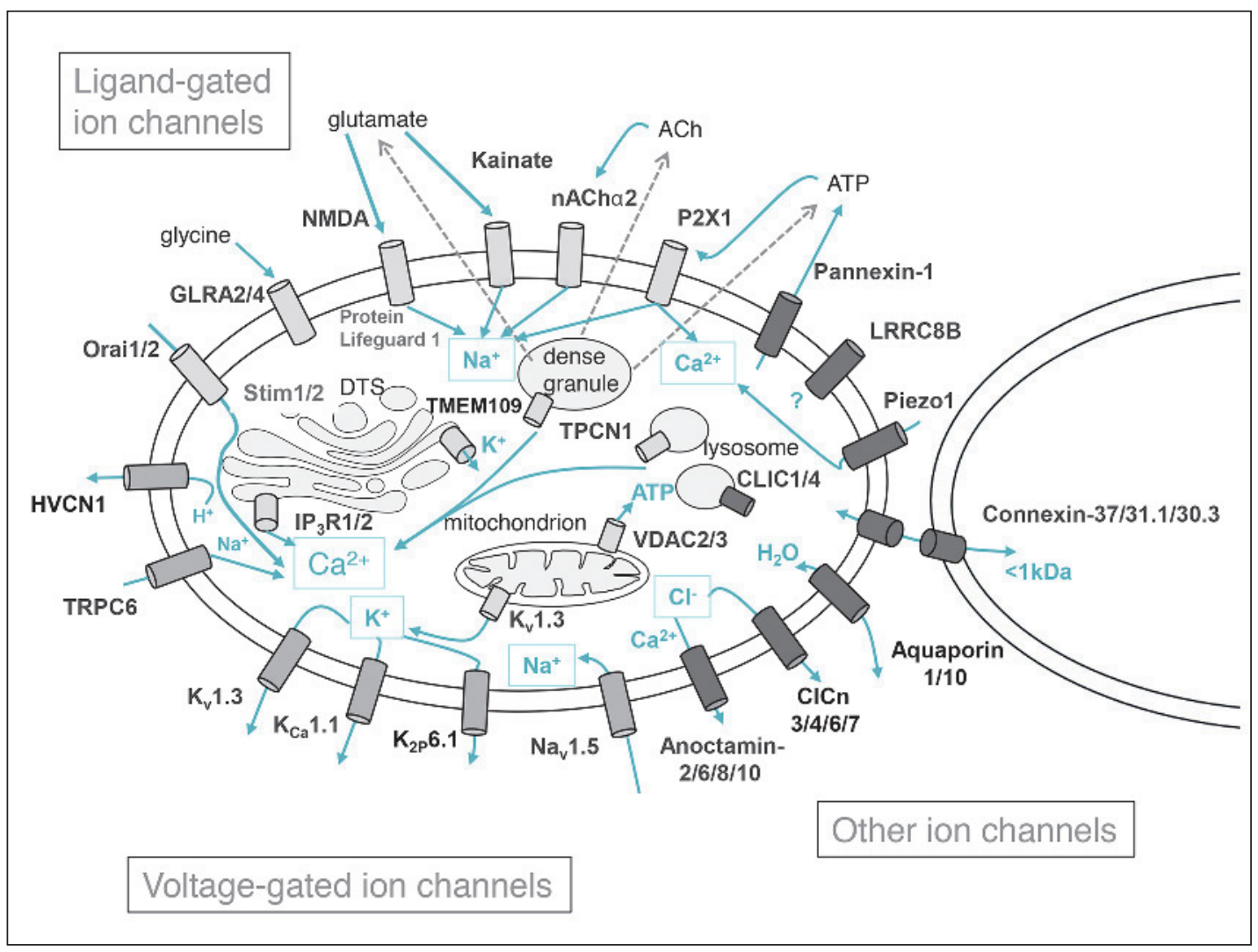

Figure 3: The human platelet channelome. A schematic summary of ion channels detected by quantitative-PCR analysis of ultrapure platelet sample preparations, classified into ligand-gated ion channels (light grey), voltage-gated ion channels (medium grey), and other ion channels (dark grey).

\section{What is known about this topic?}

- Ion channels have crucial roles in all cell types and represent important therapeutic targets.

- Approximately 20 ion channel subtypes have been reported in human platelets, and it is likely that platelets express additional members of this large family of membrane proteins.

- Megakaryocytic cell lines are frequently used as a tool to study the anuclear platelet; however, there has been no comprehensive study to compare their ion channel profile.

\section{What does this paper add?}

- This is the first study to define the human platelet ion channelome. It highlights the prominent expression of a number of ion channels that have not previously been identified in platelets and that have as yet unknown functional roles in platelet physiology and pathophysiology.

- The data provides an atlas of ion channel expression in cell-lines of megakaryoblastic/erythroleukaemic origin which may aid the selection of an appropriate surrogate cell for electrophysiological study of platelet ion channels.

- The cell-line channelome data also provides a resource that may facilitate future studies of ion channel expression in myeloid leukaemia and the identification of new therapeutic targets. cytes from patients undergoing treatment for myelogenous leukaemia (61). These findings suggest that although key cell lines provide a useful alternative tool for functional studies of the platelet, the surrogate cell must be selected with care and results must be interpreted cautiously. Therefore, our database of ion channel transcripts that are present in megakaryocytic and erythroleukaemic cell-lines provides a useful resource for selection of a suitable surrogate cell for studies of megakaryocyte ion channel function and for the study of ion channels in cancer.

In conclusion, the current study was designed to characterise the gene expression profile of the human platelet channelome. The results show that platelets express a range of different ion channels ( Figure 3), many of which have undetermined roles in this cell type. Future studies of such proteins may prove beneficial given the widespread use of ion channels as therapeutic targets and the key roles of platelets in a range of physiological and pathophysiologic processes. Although megakaryocytic cell lines have proven a useful tool for study of certain platelet proteins, the channelome datasets also highlight that such cells must be selected carefully in surrogate studies of platelet ion channels. Future investigations of the differences in ion channel expression between platelets and related leukaemic cell lines may also improve our understanding of cellular events that contribute to myeloid leukaemia. 


\section{Acknowledgements}

We acknowledge support of the British Heart Foundation (grant no PG/11/56) and the Leicester NIHR Biomedical Research Unit in Cardiovascular Disease, Glenfield Hospital, Leicester, UK. We thank Dr Conor McCloskey for assistance in compiling a list of genes within the human channelome.

\section{Author contributions}

J.R.W. designed and performed experiments, analysed data, and wrote the manuscript; S.A. designed and performed experiments, analysed data and edited the paper; A.H.G. discussed experimental design and edited the paper; and M.P.M-S. designed the project, discussed analysis and wrote the manuscript. Joy R. Wright and Stefan Amisten contributed equally to the study.

\section{Conflicts of interest}

None declared.

\section{References}

1. Franco AT, Corken A, Ware J. Platelets at the interface of thrombosis, inflammation, and cancer. Blood 2015; 126: 582-588.

2. Mahaut-Smith MP. The unique contribution of ion channels to platelet and megakaryocyte function. J Thromb Haemost 2012; 10: 1722-1732.

3. MacKenzie AB, Mahaut-Smith MP, Sage SO. Activation of receptor-operated cation channels via P2X1 not P2T purinoceptors in human platelets. J Bio Chem 1996; 271: 2879-2881.

4. Quinn M, Deering A, Stewart M, et al. Quantifying GPIIb/IIIa receptor binding using 2 monoclonal antibodies: discriminating abciximab and small molecular weight antagonists. Circulation 1999; 99: 2231-2238.

5. Hechler B, Lenain N, Marchese P, et al. A role of the fast ATP-gated P2X1 cation channel in thrombosis of small arteries in vivo. J Exp Med 2003; 198: 661-667.

6. Lewandrowski U, Wortelkamp S, Lohrig K, et al. Platelet membrane proteomics: a novel repository for functional research. Blood 2009; 114: e10-19.

7. Burkhart JM, Vaudel M, Gambaryan S, et al. The first comprehensive and quantitative analysis of human platelet protein composition allows the comparative analysis of structural and functional pathways. Blood 2012; 120 : e73-82.

8. Senis Y, Garcia A. Platelet proteomics: state of the art and future perspective. Methods Mol Biol 2012; 788: 367-399.

9. Gnatenko DV, Dunn JJ, McCorkle SR, et al. Transcript profiling of human platelets using microarray and serial analysis of gene expression. Blood 2003; 101: 2285-2293.

10. McRedmond JP, Park SD, Reilly DF, et al. Integration of proteomics and genomics in platelets: a profile of platelet proteins and platelet-specific genes. Mol Cell Proteom 2004; 3: 133-144.

11. Rowley JW, Oler AJ, Tolley ND, et al. Genome-wide RNA-seq analysis of human and mouse platelet transcriptomes. Blood 2011; 118: e101-111.

12. Bray PF, McKenzie SE, Edelstein LC, et al. The complex transcriptional landscape of the anucleate human platelet. BMC Genomics 2013; 14: 1 .

13. McCloskey C, Jones S, Amisten S, et al. Kv1.3 is the exclusive voltage-gated $\mathrm{K}+$ channel of platelets and megakaryocytes: roles in membrane potential, $\mathrm{Ca} 2+$ signalling and platelet count. J Physiol 2010; 588: 1399-1406.

14. Komatsu N. Culture of megakaryocytic cell lines: uses and limitations. Methods Mol Biol 2004; 272: 361-373.

15. Amisten S. A rapid and efficient platelet purification protocol for platelet gene expression studies. Methods Mol Biol 2012; 788: 155-172.

16. Yang $\mathrm{H}$, Kim A, David T, et al. TMEM16F forms a $\mathrm{Ca}^{2+}$-activated cation channel required for lipid scrambling in platelets during blood coagulation. Cell 2012; 151: 111-122.

17. Suzuki J, Umeda M, Sims PJ, et al. Calcium-dependent phospholipid scrambling by TMEM16F. Nature 2010; 468: 834-838.
18. Mattheij NJ, Braun A, van Kruchten R, et al. Survival protein anoctamin-6 controls multiple platelet responses including phospholipid scrambling, swelling, and protein cleavage. FASEB J 2016; 30: 727-737.

19. Liu G, Liu G, Chen $\mathrm{H}$, et al. Involvement of $\mathrm{Ca}^{2+}$ Activated $\mathrm{Cl}^{-}$Channel Ano6 in Platelet Activation and Apoptosis. Cell Physiol Biochem 2015; 37: 1934-1944.

20. Harper MT, Poole AW. Chloride channels are necessary for full platelet phosphatidylserine exposure and procoagulant activity. Cell Death Dis 2013; 4: e969.

21. Fujii T, Sakata A, Nishimura S, et al. TMEM16F is required for phosphatidylserine exposure and microparticle release in activated mouse platelets. Proc Natl Acad Sci USA 2015; 112: 12800-12805.

22. Littler DR, Harrop SJ, Goodchild SC, et al. The enigma of the CLIC proteins: Ion channels, redox proteins, enzymes, scaffolding proteins? FEBS Lett 2010; 584: 2093-2101.

23. Qiu MR, Jiang L, Matthaei KI, et al. Generation and characterization of mice with null mutation of the chloride intracellular channel 1 gene. Genesis 2010; 48: 127-136.

24. Jentsch TJ. CLC chloride channels and transporters: from genes to protein structure, pathology and physiology. Crit Rev Biochem Mol Biol 2008; 43: 3-36.

25. Usman H, Mathew MK. Potassium channel regulator KCNRG regulates surface expression of Shaker-type potassium channels. Biochem Biophys Res Commun 2010; 391: 1301-1305.

26. Seddik R, Jungblut SP, Silander OK, et al. Opposite effects of KCTD subunit domains on GABA(B) receptor-mediated desensitization. J Biol Chem 2012; 287: 39869-39877.

27. Hassock SR, Zhu MX, Trost C, et al. Expression and role of TRPC proteins in human platelets: evidence that TRPC6 forms the store-independent calcium entry channel. Blood 2002; 100: 2801-2811.

28. Harper MT, Londono JE, Quick K, et al. Transient receptor potential channels function as a coincidence signal detector mediating phosphatidylserine exposure. Science Signal 2013; 6: ra50.

29. Zbidi H, Lopez JJ, Amor NB, et al. Enhanced expression of STIM1/Orail and TRPC3 in platelets from patients with type 2 diabetes mellitus. Blood Cells Mol Dis 2009; 43: 211-213.

30. Carter RN, Tolhurst G, Walmsley G, et al. Molecular and electrophysiological characterization of transient receptor potential ion channels in the primary murine megakaryocyte. J Physiol 2006; 576: 151-162.

31. Pottosin I, Delgado-Enciso I, Bonales-Alatorre E, et al. Mechanosensitive $\mathrm{Ca}^{2+}$-permeable channels in human leukemic cells: pharmacological and molecular evidence for TRPV2. Biochim Biophys Acta 2015; 1848: 51-59.

32. Tolhurst G, Carter RN, Amisten S, et al. Expression profiling and electrophysiological studies suggest a major role for Orail in the store-operated $\mathrm{Ca}^{2+}$ influx pathway of platelets and megakaryocytes. Platelets 2008; 19: 308-313.

33. Braun A, Varga-Szabo D, Kleinschnitz C, et al. Orail (CRACM1) is the platelet SOC channel and essential for pathological thrombus formation. Blood 2009; 113: 2056-2063.

34. Gilio K, van Kruchten R, Braun A, et al. Roles of platelet STIM1 and Orail in glycoprotein VI- and thrombin-dependent procoagulant activity and thrombus formation. J Biol Chem 2010; 285: 23629-23638.

35. Brandman O, Liou J, Park WS, et al. STIM2 is a feedback regulator that stabilizes basal cytosolic and endoplasmic reticulum $\mathrm{Ca}^{2+}$ levels. Cell 2007; 131: 1327-1339.

36. Takeshima H, Venturi E, Sitsapesan R. New and notable ion-channels in the sarcoplasmic/endoplasmic reticulum: do they support the process of intracellular $\mathrm{Ca}^{2+}$ release? J Physiol 2015; 593: 3241-3251.

37. Calcraft PJ, Ruas M, Pan Z, et al. NAADP mobilizes calcium from acidic organelles through two-pore channels. Nature 2009; 459: 596-600.

38. Vial C, Hechler B, Leon C, et al. Presence of P2X1 purinoceptors in human platelets and megakaryoblastic cell lines. Thromb Haemost 1997; 78: 1500-1504.

39. Zhao J, Ennion SJ. Sp1/3 and NF-1 mediate basal transcription of the human P2X1 gene in megakaryoblastic MEG-01 cells. BMC Mol Biol 2006; 7: 10.

40. Kalev-Zylinska ML, Green TN, Morel-Kopp MC, et al. N-methyl-D-aspartate receptors amplify activation and aggregation of human platelets. Thrombosis Res 2014; 133: 837-847.

41. Morrell CN, Sun H, Ikeda M, et al. Glutamate mediates platelet activation through the AMPA receptor. J Exp Med 2008; 205: 575-584.

42. Walther DJ, Peter JU, Winter S, et al. Serotonylation of small GTPases is a signal transduction pathway that triggers platelet alpha-granule release. Cell 2003; 115: 851-862. 
43. Schemmer P, Zhong Z, Galli U, et al. Glycine reduces platelet aggregation. Amino Acids 2013; 44: 925-931.

44. Vaiyapuri S, Moraes LA, Sage T, et al. Connexin 40 regulates platelet function. Nature Commun 2013; 4: 2564.

45. Angelillo-Scherrer A, Fontana P, Burnier L, et al. Connexin 37 limits thrombus propensity by downregulating platelet reactivity. Circulation 2011; 124: 930-939.

46. Sosinsky GE, Boassa D, Dermietzel R, et al. Pannexin channels are not gap junction hemichannels. Channels 2011; 5: 193-197.

47. Taylor KA, Wright JR, Vial C, et al. Amplification of human platelet activation by surface pannexin-1 channels. J Thromb Haemost 2014; 12: 987-998.

48. Abascal F, Zardoya R. LRRC8 proteins share a common ancestor with pannexins, and may form hexameric channels involved in cell-cell communication. BioEssays 2012; 34: 551-560.

49. Voss FK, Ullrich F, Munch J, et al. Identification of LRRC8 heteromers as an essential component of the volume-regulated anion channel VRAC. Science 2014; 344: 634-638.

50. Albuisson J, Murthy SE, Bandell M, et al. Dehydrated hereditary stomatocytosis linked to gain-of-function mutations in mechanically activated PIEZO1 ion channels. Nature Commun 2013; 4: 1884.

51. DeCoursey TE. Voltage-gated proton channels: molecular biology, physiology, and pathophysiology of the H(V) family. Physiol Rev 2013; 93: 599-652.

52. Carrithers MD, Dib-Hajj S, Carrithers LM, et al. Expression of the voltage-gated sodium channel NaV1.5 in the macrophage late endosome regulates endosomal acidification. J Immunol 2007; 178: 7822-7832.

53. Oury C, Toth-Zsamboki E, Thys C, et al. The ATP-gated P2X1 ion channel acts as a positive regulator of platelet responses to collagen. Thromb Haemost 2001 86: 1264-1271.

54. Rolf MG, Brearley CA, Mahaut-Smith MP. Platelet shape change evoked by selective activation of P2X1 purinoceptors with alpha,beta-methylene ATP Thromb Haemost 2001; 85: 303-308.

55. Londin ER, Hatzimichael E, Loher P, et al. The human platelet: strong transcriptome correlations among individuals associate weakly with the platelet proteome. Biology Direct 2014; 9: 3.

56. Rowley JW, Weyrich AS. Coordinate expression of transcripts and proteins in platelets. Blood 2013; 121: 5255-5256.

57. Nagaraj N, Wisniewski JR, Geiger T, et al. Deep proteome and transcriptome mapping of a human cancer cell line. Mol Systems Biol 2011; 7: 548 .
58. Kim MS, Pinto SM, Getnet D, et al. A draft map of the human proteome. Nature 2014; 509: 575-581.

59. Mahaut-Smith MP. Calcium-activated potassium channels in human platelets. J Physiol 1995; 484: 15-24.

60. Stoneking CJ, Shivakumar O, Thomas DN, et al. Voltage dependence of the $\mathrm{Ca}^{2+}$-activated $\mathrm{K}^{+}$channel $\mathrm{K}(\mathrm{Ca}) 3.1$ in human erythroleukemia cells. Am J Physiol Cell Physiol 2013; 304: C858-872.

61. Kapural L, Feinstein MB, O'Rourke F, et al. Suppression of the delayed rectifier type of voltage gated $\mathrm{K}+$ outward current in megakaryocytes from patients with myelogenous leukemias. Blood 1995; 86: 1043-1055.

62. Agbani EO, van den Bosch MT, Brown E, et al. Coordinated Membrane Ballooning and Procoagulant Spreading in Human Platelets. Circulation 2015; 132 1414-1424.

63. Oury C, Kuijpers MJ, Toth-Zsamboki E, et al. Overexpression of the platelet $\mathrm{P} 2 \mathrm{X} 1$ ion channel in transgenic mice generates a novel prothrombotic phenotype. Blood 2003; 101: 3969-3976.

64. Molica F, Morel S, Meens MJ, et al. Functional role of a polymorphism in the Pannexinl gene in collagen-induced platelet aggregation. Thromb Haemost 2015; 114: 325-336.

65. Quinton TM, Dean WL. Multiple inositol 1,4,5-trisphosphate receptor isoforms are present in platelets. Biochem Biophys Res Commun 1996; 224: 740-746.

66. Varga-Szabo D, Braun A, Kleinschnitz C, et al. The calcium sensor STIM1 is an essential mediator of arterial thrombosis and ischemic brain infarction. J Exp Med 2008; 205: 1583-1591.

67. Grosse J, Braun A, Varga-Szabo D, et al. An EF hand mutation in Stiml causes premature platelet activation and bleeding in mice. J Clin Invest 2007; 117: 3540-3550.

68. Ramanathan G, Gupta S, Thielmann I, et al. Defective diacylglycerol-induced $\mathrm{Ca}^{2+}$ entry but normal agonist-induced activation responses in TRPC6-deficient mouse platelets. J Thromb Haemost 2012; 10: 419-429.

69. Vemana HP, Karim ZA, Conlon C, et al. A critical role for the transient receptor potential channel type 6 in human platelet activation. PloS ONE 2015; 10: e0125764.

70. El-Daher SS, Patel Y, Siddiqua A, et al. Distinct localization and function of $(1,4,5) \operatorname{IP}(3)$ receptor subtypes and the $(1,3,4,5) \operatorname{IP}(4)$ receptor GAP1(IP4BP) in highly purified human platelet membranes. Blood 2000; 95: 3412-3422.

71. Mahaut-Smith MP, Rink TJ, Collins SC, et al. Voltage-gated potassium channels and the control of membrane potential in human platelets. J Physiol 1990; 428: 723-735. 